Immobilization of monolayer protected lipophilic gold nanorods on a glass surface

This article has been downloaded from IOPscience. Please scroll down to see the full text article.

2012 Nanotechnology 23055605

(http://iopscience.iop.org/0957-4484/23/5/055605)

View the table of contents for this issue, or go to the journal homepage for more

Download details:

IP Address: 155.185.228.120

The article was downloaded on 19/01/2012 at 15:27

Please note that terms and conditions apply. 


\title{
Immobilization of monolayer protected lipophilic gold nanorods on a glass surface
}

\author{
Guido Ori $^{1}$, Denis Gentili ${ }^{2,3}$, Massimiliano Cavallini ${ }^{2}$, \\ Mauro Comes Franchini ${ }^{3}$, Mauro Zapparoli ${ }^{4}$, Monia Montorsi ${ }^{1}$ and \\ Cristina Siligardi ${ }^{1}$
}

\author{
${ }^{1}$ Dipartimento di Ingegneria dei Materiali e dell'Ambiente, Università di Modena e Reggio Emilia, \\ Via Vignolese 905/A, 41100 Modena, Italy \\ 2 CNR-ISMN Istituto per lo Studio dei Materiali Nanostrutturati, Via P Gobetti 101, I-40129 Bologna, \\ Italy \\ ${ }^{3}$ Dipartimento di Chimica Organica ‘A Mangini’, Università di Bologna, Viale Risorgimento, Bologna, \\ Italy \\ ${ }^{4}$ Centro Interdipartimentale Grandi Strumenti (CIGS), Via Campi 213/A, 41100 Modena, Italy \\ E-mail: guido.ori@unimore.it and d.gentili@bo.ismn.cnr.it
}

Received 22 September 2011, in final form 14 November 2011

Published 11 January 2012

Online at stacks.iop.org/Nano/23/055605

\begin{abstract}
We present a novel process of immobilization of gold nanorods (GNRs) on a glass surface. We demonstrate that by exploiting monolayer protection of the GNRs, their unusual optical properties can be completely preserved. UV-visible spectroscopy and atomic force microscopy analysis are used to reveal the optical and morphological properties of monolayer protected immobilized lipophilic GNRs, and molecular dynamics simulations are used to elucidate their surface molecule arrangements.

S Online supplementary data available from stacks.iop.org/Nano/23/055605/mmedia
\end{abstract}

(Some figures may appear in colour only in the online journal)

\section{Introduction}

Gold nanorods (GNRs) are promising nanostructures because of their unique, fascinating and tunable anisotropic optical and physical properties; for example, the longitudinal plasmon resonance (LPR) band of GNRs can be tuned over the visible to near infrared (NIR) wavelength range by changing their aspect ratio (the ratio of length to width) [1-3]. Because solid-state (glass-based) devices with unusual and customized optical functionalities are sought, such properties are important for a number of specific technological applications, such as in providing decorative coatings [4], catalysts [5], optical filters [6, 7], and nonlinear optical materials [8], and other functional applications [1-3].

So far, GNRs have been mainly prepared and functionalized in aqueous solutions [9] and only a few methods have been proposed for transferring GNRs in organic media, to obtain organo-soluble (lipophilic) GNRs with completely preserved optical properties [10-13]. Lipophilic GNRs have been successfully prepared by means of polymer/polyelectrolyte coatings [10, 13], but only a few studies have reported the thiol-based monolayer protection of
GNRs as an elegant easily used tool for transferring GNRs in organic solvent (not miscible with water) [11, 12]. In general, monolayer protection of lipophilic gold nanoparticles (NPs) gives superior stability, lower interfacial energy and better compatibility with organic polymers and surfaces in comparison with water-soluble NPs, opening the way to a high degree of control during solution and surface processing, as well as greater functionality [12, 14].

Several approaches using aqueous GNRs have been developed for achieving nanostructured GNR patterns on solid supports [6, 15]. Although these nanostructured materials have potential uses in areas ranging from electronics to biosensors [16], after deposition of the GNRs their optical properties are found to be substantially changed. It seems reasonable to expect the monolayer protection of lipophilic GNRs to open the way to a wide spectrum of innovative specific applications, because of the opportunity that it affords for customizing desirable further molecular functionalization of GNRs directly or on solid-state supports.

In light of this, with the aim of transferring the unusual optical properties of GNRs on a solid-state support, here we report a new approach for the immobilization of GNRs on a 
thiol-functionalized glass surface, highlighting the importance of the GNR ligand shell, time of incubation and GNR concentration.

\section{Materials and methods}

All chemicals were purchased from Sigma-Aldrich (St Louis, MO) and used as received; they included cetyltrimethylammonium bromide, CTAB H6269 (99\%), and 3-mercaptopropyltrimethoxysilane, MPTS (95\%).

Following the methods of El-Sayed and co-workers, the GNRs were prepared by the seed-mediated growth method, with different aspect ratios $\left(1 \mathrm{mg} \mathrm{ml}^{-1}\right.$ gold and 10 mM CTAB) [9]. Lipophilic GNRs were obtained by the simple one-step phase transfer process [11], which is based on a straightforward one-step ligand exchange reaction in a hydro-alcoholic mixture with 1-thiol $\left(1 \mathrm{mg} \mathrm{ml}^{-1}\right.$ gold and $2 \mathrm{mM}$ 1-thiol). MPTS-functionalized glass slides $(2 \times$ $1 \mathrm{~cm}^{2}$; see ESI, available at stacks.iop.org/Nano/23/055605/ mmedia) were then dipped, in the vertical position to exclude physical deposition on them, into GNR solutions of different concentrations and for different times $(30 \mathrm{~min}, 2 \mathrm{~h}$ and overnight). The GNR solutions used for the immobilization on glass slides were aqueous GNR-CTAB solution and lipophilic GNR-1 solution, obtained by the 1:10 dilution $\left(0.1 \mathrm{mg} \mathrm{ml}^{-1}\right.$ gold, labeled as $\mathbf{C}$, for concentrated) and 1:30 dilution $\left(0.034 \mathrm{mg} \mathrm{ml}^{-1}\right.$ gold, labeled as $\mathbf{D}$, for diluted) of the corresponding solution obtained from the synthesis. After the immobilization step, the glass slides were washed several times with water (for GNR-CTAB) or chloroform (for GNR-1) and with ethanol, and then dried in a stream of $\mathrm{N}_{2}$.

\section{Results and discussion}

The water-soluble GNRs (GNR-CTAB) with different aspect ratios employed in our experiments were all prepared by a seed-mediated growth method [9] using a high concentration of the cationic quaternary ammonium surfactant cetyltrimethylammonium bromide (CTAB; figure 1) as the stabilizing agent. The synthesis was scaled up to volumes over $100 \mathrm{ml}$ without modification. The original concentration of CTAB (100 mM) was sequentially lowered to $10 \mathrm{mM}$ by successive centrifugations and re-suspensions in DI water. Figures 2(A) and (B) show a representative UV-visible absorption spectrum and a representative TEM image of GNR-CTAB with an aspect ratio of $\sim 4.4$ (average size of $\sim 61 \mathrm{~nm} \pm 5 \mathrm{~nm} \times 14 \pm 2 \mathrm{~nm}$ ) whose LPR and transverse plasmon resonance (TPR) bands are located at 784 and $509 \mathrm{~nm}$, respectively. The former band dominates the profile of the spectrum as compared with the TPR band, confirming the low concentration of by-products (as spherical nanoparticles).

In order to study the importance of the ligand shell of the GNRs as regards their immobilization on a thiolfunctionalized glass surface, lipophilic CTAB-free GNRs have been obtained by a straightforward one-step ligand exchange reaction with ligand 1 (figure 1 ) of freshly prepared GNR-CTAB in a hydro-alcoholic mixture [11]. In this

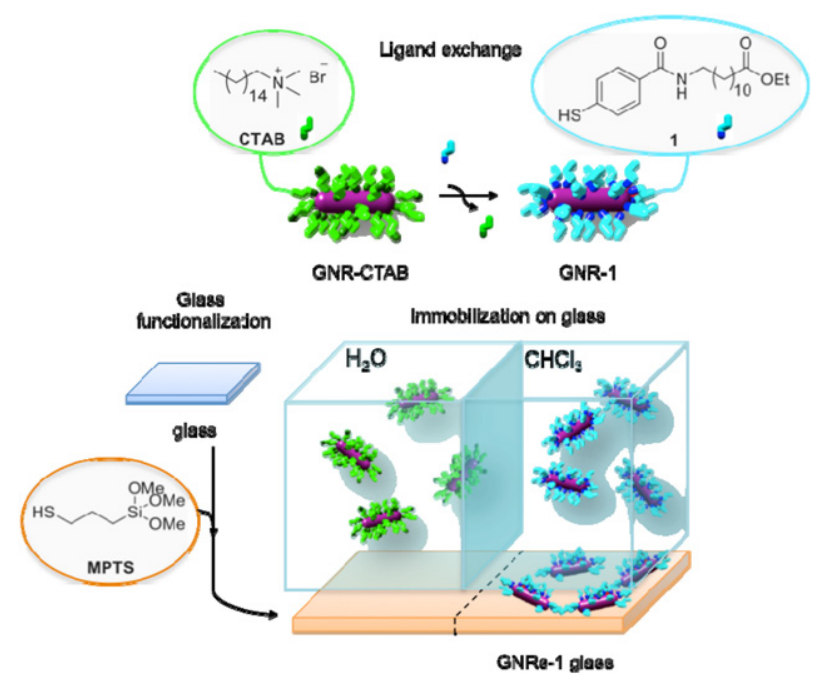

Figure 1. Chemical structures of the ligands used to functionalize GNRs (CTAB and 1) and glass (MPTS) surfaces, and a schematic representation of the GNR ligand exchange and of the immobilization of GNRs on a glass surface.

way, GNRs are coated with a monolayer of $\mathbf{1}$ (GNR-1), which stabilizes them in chloroform solution, preserving their unusual optical properties. The absorption profile spectrum (figure 2(A)), where the LPR band of GNR-1 is red shifted due to the change of refractive index of the surrounding environment [11], confirms the absence of aggregations, revealing no broadening and shape variation.

The TEM image of GNR-1 (figure 2(C)) shows no significant size and shape changes of the GNRs and a ring-like organization, revealing the hydrophobic nature of their surface [17], which is further confirmed by photographs shown in figure 2(D). The FTIR spectrum (figure 3) of GNR-1 shows the absence of the quaternary amine stretch of CTAB $\left(958 \mathrm{~cm}^{-1}\right)$ [11] and the presence of peaks of ligand $\mathbf{1}$ in particular, NH deformation combined with a CN stretch (1599 and $\left.1515 \mathrm{~cm}^{-1}\right)$ and an amide $\mathrm{C}=\mathrm{O}$ stretch $\left(1673 \mathrm{~cm}^{-1}\right)$ shifted and partially overlapped with an ester $\mathrm{C}=\mathrm{O}$ stretch $\left(1737 \mathrm{~cm}^{-1}\right)$.

Molecular dynamics simulations of the ligand surface morphology on metal surfaces have been demonstrated to be a useful complement to experiments for obtaining insight into ligand-metal surface interactions [14, 18-21]. Herein, MD simulation of $1 \mathrm{SAM}$ on $\mathrm{Au}(111)$ surface has been used to obtain insight into the surface morphology of 1 SAM. MD simulation of 1 SAM showed typical oriented thiol SAM arrangements, showing an angular distribution of the tilted angle centered on $\sim 30.5^{\circ}$ (averaged between the phenyl and alkyl tilt angles; see the supporting material available at stacks.iop.org/Nano/23/055605/mmedia), in good accordance with literature values $\left(\sim 30^{\circ}\right)$ [22]. Furthermore, the results from MD simulation demonstrate the formation of a strong network of $\mathrm{H}$ bonds between amide groups and $\mathbf{1}$ molecules, oriented parallel to the gold surface (figure 3(B)) [23-25]. This confirms the importance of the design of the ligand as regards providing improved robustness for the GNR ligand shell [11]. The thickness of the 1 SAM was determined by atomic density profile (ADP) analysis of the system; 


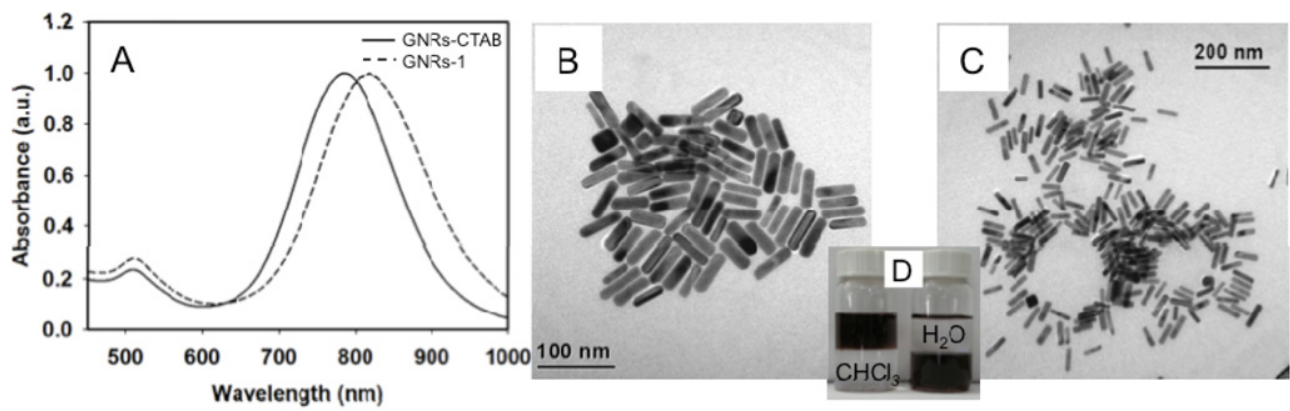

Figure 2. Representative UV-visible spectra (A) and TEM images ((B) and (C)) of water-soluble (GNR-CTAB) and lipophilic (GNR-1) gold nanorods, respectively. (D) Photographs of GNRs dissolved in water (GNR-CTAB) and in chloroform (GNR-1) after ligand exchange.
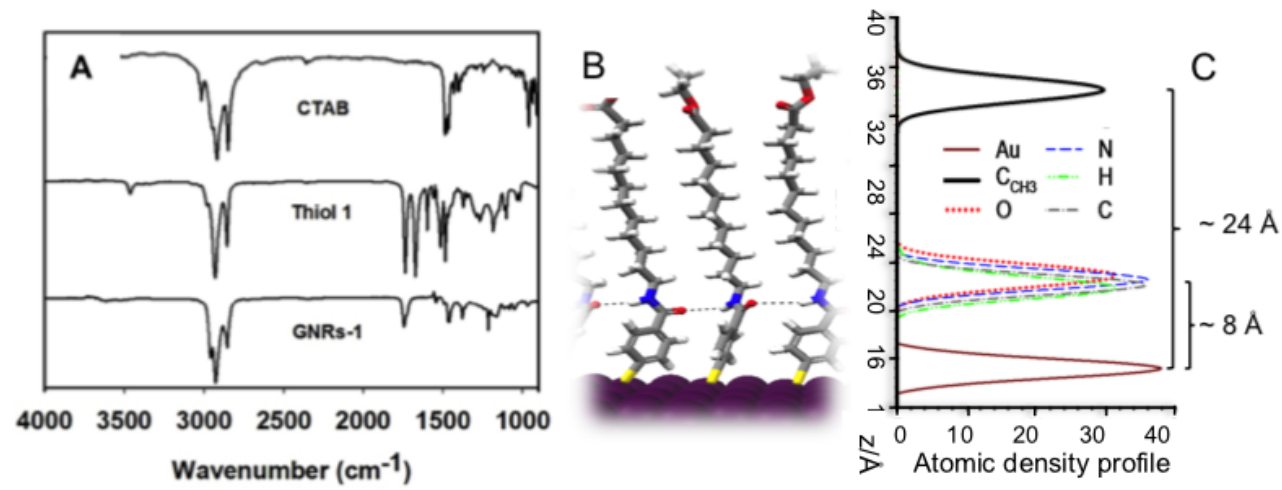

Figure 3. (A) FTIR spectra of CTAB (KBr), neat $1\left(\mathrm{CCl}_{4}\right)$ and GNR-1. (B) MD snapshot of $\mathbf{1}$ SAM (self-assembled monolayers) on an $\mathrm{Au}(111)$ surface showing the intermolecular $\mathrm{H}$ bonding between 1 molecules (black dashed line; Au: violet, S: yellow, C: gray, N: blue, O: red, $\mathrm{H}$ : white). (C) Atomic density profile of Au surface atoms and $\mathrm{O}, \mathrm{N}, \mathrm{H}$ and $\mathrm{C}$ atoms constituting the amide group and $\mathrm{C}$ from the external methyl group.

the value obtained $(\sim 24 \AA)$ is in quite good accordance with ellipsometry and XPS data for thiols with comparable size [26]. Moreover, ADP analysis of the O, N, H, and $\mathrm{C}$ constituting the 1-amide group units showed averaged interatomic distances with respect to $\mathrm{Au}$ surface atoms of $\sim 8 \AA$. In light of this, the difference between the FTIR spectra of 1 monomer and GNR-1 (especially in the 1500-1750 $\mathrm{cm}^{-1}$ range) could be ascribed to the vicinity with the gold surface and probably also the presence of the phenyl ring; both could affect the $\mathrm{H}$ bonding network promoted by the $\mathbf{1}$-amide group units. This is in part in accordance with previously reported studies on gold nanoparticle/planar surfaces and further investigation will be necessary to understand these effects [22-27].

With the aim of immobilizing GNRs through the formation of sulfur-gold bonds, the glass surface has been functionalized with 3-mercaptopropyltrimethoxysilane (MPTS; see figure 1). In accordance with previous reports [28, 29], by co-condensation of the methoxy groups of the MPTS with the hydroxyl groups of the glass surface, a highly ordered and thiol-functionalized glass surface has been achieved, as confirmed by the contact angle [30] and AFM (roughness analysis) [31].

Mercaptosilane-modified glass substrates were immersed in GNR-CTAB aqueous solutions and GNR-1 chloroform solutions to investigate the effect of three factors: the incubation time (30 min, $2 \mathrm{~h}$ or overnight); the ligand shell of the GNRs (CTAB versus ligand 1); and the concentration of GNRs (C (concentrated) solution, $0.1 \mathrm{mg} \mathrm{ml}^{-1}$ gold, versus $\mathbf{D}$ (diluted) solution, $0.034 \mathrm{mg} \mathrm{ml}^{-1}$ gold). These values (for $\mathbf{C}$ and $\mathbf{D}$ solutions) have been selected in order to show two concentration limits which correspond to two different representative situations that can be readily differentiated through experimental characterization (UV and AFM). Contact angle (CA) and AFM measurements have been used to investigate and monitor the process of immobilization of GNRs on the glass surface, while the optical properties of GNRs on solid-state supports have been studied by UV-visible absorption spectroscopy.

In contrast with previous findings [32], in our experimental conditions, water-soluble GNR-CTAB showed no propensity to be immobilized on the glass surfaces. This behavior could be due to the high concentration of CTAB in the water-soluble GNR solutions (both concentrated and diluted) used, which is a condition necessary for preserving GNR stability in solution but hinders the interaction of GNRs with the glass surface [33]. In contrast, lipophilic GNR-1 showed a clear tendency to be immobilized on the mercaptosilane-modified glass surfaces. Monolayer functionalization, as well as avoiding the crystallization of ligand excess, makes the GNRs in GNR-1 more readily available for immobilization compared with those in GNR-CTAB underlining the usefulness and the potentialities of monolayer protected nanoparticles [14]. Figure 4 shows UV-visible absorption spectra of the starting lipophilic GNR-1 (solution) and of the GNR-1 coated glasses (the solid-state form). 


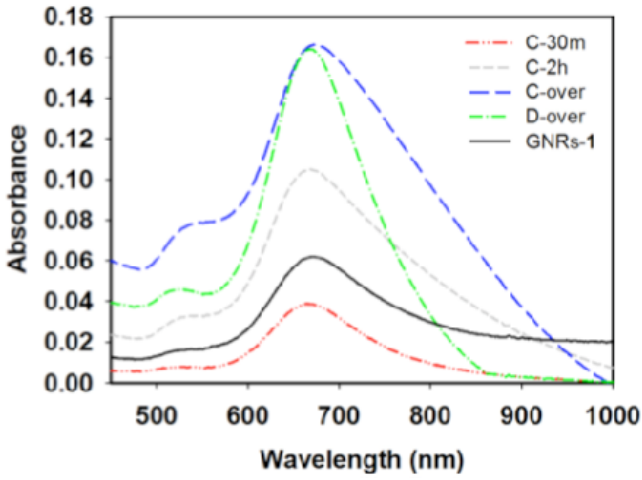

Figure 4. UV-visible spectra of the starting GNR-1 (reduced by a factor of 10 for the purposes of clarity) and of GNR-1 immobilized on glass slides under different conditions.

UV-visible spectra confirm the immobilization of the GNR-1 on the glass surface for all the samples prepared, independently of time of incubation and GNR concentration. In detail, looking at the samples obtained by incubation with concentrated GNR-1 solution (C samples), we see that the absorption of GNR-1 coated glass increases with the time of incubation. This trend is stronger at the LPR maximum where the absorbance after 30 min of incubation ('C-30 min') is $\sim 0.04$, increasing to $\sim 0.10$ after $2 \mathrm{~h}$ of incubation ('C-2 h') and reaching $\sim 0.16$ after incubation overnight ('C-over'). No further increase of absorption was noted after the overnight incubation. Looking at the full width at half-maximum (FWHM) of the LPR band, a slight propensity to form aggregates of GNRs over time can be observed. The high concentration of GNRs probably induces instability in the system in the presence of mercaptosilane-modified glass surface, which leads to the formation of small GNR agglomerates before their immobilization on the glass surface. Furthermore, the position of the $\mathrm{LPR}_{\max }$ band changed from 664 to $672 \mathrm{~nm}$ with increasing time of incubation, becoming quite close to the LPR band position for the GNR-1 in chloroform solution $\left(\lambda_{\mathrm{LPR}}=673 \mathrm{~nm}\right)$. This is probably related to the change of refractive index of the medium surrounding the GNRs [23, 34], but further investigation will be necessary to achieve a detailed understanding of this aspect. Likewise, the UV-visible absorption profile confirms the immobilization of GNR-1 on glass substrates by incubation overnight in diluted GNR-1 solution (the D-over sample). In particular, the LPR band absorption profiles for D samples appear narrower (with smaller FWHM) than those for all the previous $\mathbf{C}$ samples, showing a blue-shifted LPR band at $667 \mathrm{~nm}$. This indicates that with a diluted solution, the aggregation of GNR-1 can be reduced or completely avoided, with the optical properties of GNR-1 transferring completely to the final solid-state device. Also in this case, no further increase of absorption has been noted by UV-visible spectroscopy, indicating a possible complete saturation of the thiol groups on the glass surface without affecting the UV-visible properties of GNR-1.

After silane surface functionalization with MPTS, water static contact angle measurements proved a decrease in the hydrophilicity of the glass surface (see ESI, S1,

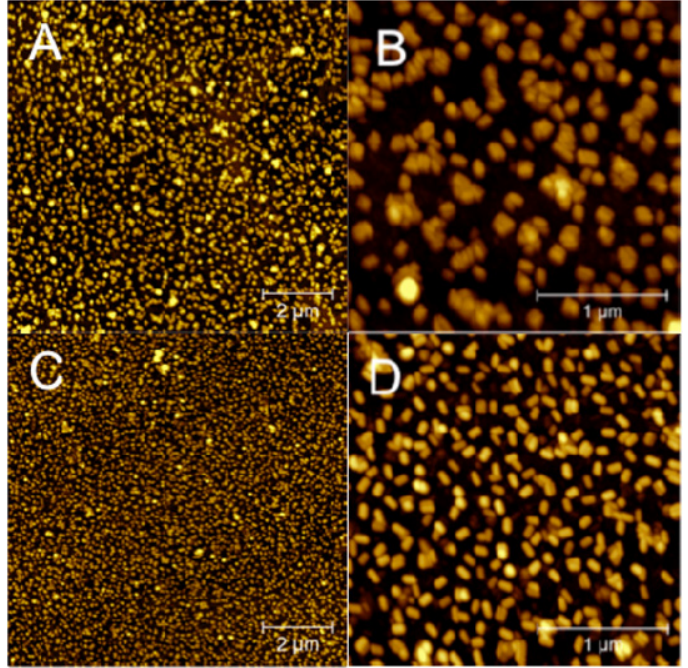

Figure 5. 2D AFM images of $\mathbf{C}$-over ((A) and (B)) and D-over $((\mathrm{C})$ and (D)) GNR-1 coated glasses $\left(\mathrm{A} / \mathrm{C}=10 \times 10 \mu \mathrm{m}^{2}\right.$ and $\mathrm{B} / \mathrm{D}=2.5 \times 2.5 \mu \mathrm{m}^{2}$ ).

available at stacks.iop.org/Nano/23/055605/mmedia), due to the hydrolysis and condensation of the silane which orient the molecule with the thiol group extended normal to the surface. After incubation with GNR-1 solutions, independently of the incubation conditions tested, glass substrates show no significant CA variations as compared with MPTS glass. This indicates that GNR-1 surface coatings show similar surface energies to the MPTS-functionalized glass, which underlines the lack of evidence of 3D GNR agglomeration on the glass surface which could significantly alter the roughness and consequently the wettability of the glass surface.

Analysis of AFM images allows us to obtain further insight into the surface morphologies of the GNR coated glass. In particular, we further confirmed the absence of aqueous GNR-CTAB on the glass surface for all conditions tested, while lipophilic GNR-1 tends to attach to the glass, lying along the long axis. In the main, AFM micrographs show that GNR-1 tends to show side-by-side aggregation rather than tail-to-tail aggregation, in a flat configuration maximizing the surface contact with the MPTS glass. Figure 5 shows the disposition of GNR-1 immobilized on C-over and $\mathbf{D}$-over samples at different magnifications. Comparing the morphologies, it is evident that on increasing the concentration of GNR-1 solution we move from nearly isolated GNR-1 to clusters (with $\sim 4-10$ GNRs), which is in full agreement with the previous observations made from UV-visible spectrum profiles. Therefore, the remarkable preservation of UV-visible absorption properties in D-over samples can be attributed to the discrete space that separates single GNR-1 units. The height of immobilized GNR-1 has been measured statistically from the height distribution graph (figure 6), in which the difference $(\sim 17 \mathrm{~nm})$ between the positions of the peaks is the average height of the immobilized GNR-1. Such values, which are similar for the $\mathbf{C}$-over and $\mathbf{D}$-over samples, demonstrate the achieving of a monolayer GNR-1 coating, indicating that only the GNRs directly in contact with the functionalized surface can be 


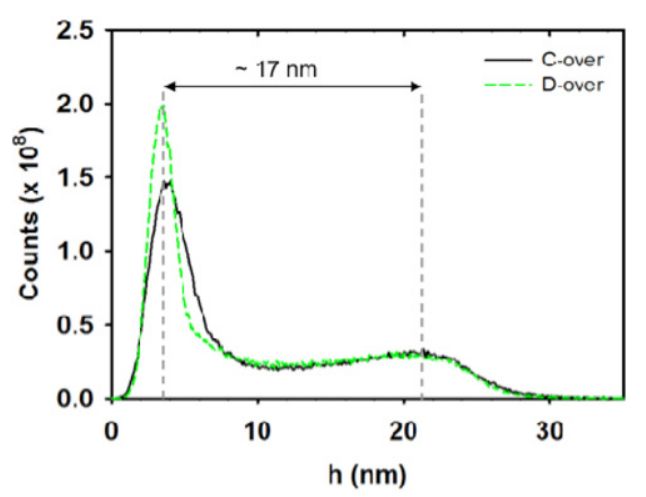

Figure 6. Height distribution graph of the topographic images of C-over and D-over GNR-1 coated glasses.

immobilized. D and $\mathbf{C}$ samples show similar roughnesses (RMS) of $7.6 \pm 0.3 \mathrm{~nm}$ and $7.9 \pm 0.2 \mathrm{~nm}$, respectively. As expected, these data are increased in comparison with those for mercaptosilane-modified glass $(\sim 0.55 \mathrm{~nm})$ and this further confirms the presence of a single layer of GNR-1 over the glass surface. In light of this, considering that $\mathbf{D}$ and $\mathbf{C}$ samples show similar UV-visible absorption intensity at the maximum of the LPR band, the numbers of GNR-1 units on the surface must be nearly the same, as is indeed confirmed by calculation of the percentage of the surface covered by GNR-1, which is, for both samples, $~ 35 \%$.

\section{Conclusions}

In conclusion, our results clearly demonstrate the feasibility of immobilizing GNRs on a solid support such as a thiolfunctionalized glass slide, preserving their unusual optical properties. This result is made possible using lipophilic GNR-1, obtained by changing the surface chemistry of GNRs in a well-defined manner. Using MD simulations, further insight into the thiol molecule arrangement on a gold surface has been obtained, highlighting the strong $\mathrm{H}$ network promoted in the $1 \mathrm{SAM}$. Understanding how to manipulate GNRs on solid-state supports (such as glass surfaces) opens the way to a wide spectrum of potential multifunctional applications in materials science, on the basis of their unusual and customizable properties.

\section{Acknowledgments}

Dr F Terzi (University of Modena, Italy) is acknowledged for helpful discussion on FTIR data. Dr C Albonetti (CNR-ISMN, Bologna, Italy) is acknowledged for helpful discussion on AFM data. DG and MC were supported by ESF-EURYI DYMOT.

\section{References}

[1] Murphy C J, Gole A M, Stone J W, Sisco P N, Alkilany A M, Goldsmith E C and Baxter S C 2008 Acc. Chem. Res. 41 1721-30
[2] Jain P K, Huang X, El-Sayed I H and El-Sayed M A 2008 Acc. Chem. Res. 41 1578-86

[3] Tong L, Wei Q, Wei A and Cheng J-X 2009 Photochem. Photobiol. 85 21-32

[4] Weyl W A 1951 Coloured Glasses vol 23 (Sheffield: Society of Glass Technology)

[5] Braun S, Rappoport S, Zusman R, Avnir D and Ottolenghi M 1990 Mater. Lett. 10 1-5

[6] Xu X, Gibbons T H and Cortie M B 2006 Gold Bull. 39 156-65

[7] Böhmer M R, Balkenende A R, Bernards T N M, Peeters M P J, van Bommel M J, Boonekamp E P, Verheijen M A, Krings L H M and Vroon Z A E P 2001 Handbook of Advanced Electronic and Photonic Devices vol 5 (San Diego, CA: Academic)

[8] Fukumi K, Chayahara A, Kadono K, Sakaguchi T, Horino Y, Miya M, Fujii K, Hayakawa J and Satou M 1994 J. Appl. Phys. 75 3075-80

[9] Nikoobakht B and El-Sayed M A 2003 Chem. Mater. 15 1957-62

[10] Alkilany A M, Thompson L B and Murphy C J 2010 ACS Appl. Mater. Interfaces 2 3417-21

[11] Gentili D, Ori G and Franchini M C 2009 Chem. Commun. $5874-6$

[12] Li Y, Yu D, Dai L, Urbas A and Li Q 2011 Langmuir 27 98-103

[13] Thierry B, Ng J, Krieg T and Griesser H J 2009 Chem. Commun. 1 1724-6

[14] Hung A, Mwenifumbo S, Mager M, Kuna J J, Stellacci F, Yarovsky I and Stevens M M $2011 \mathrm{~J}$. Am. Chem. Soc. 133 1438-50

[15] Yi D K, Lee J H, Rogers J A and Paik U 2009 Appl. Phys. Lett. 94084104

[16] Stebe K J, Lewandowski E and Ghosh M 2009 Science 325159

[17] Khanal B P and Zubarev E R 2007 Angew. Chem. Int. Edn $462195-8$

[18] Goujon F, Bonal C, Limoges B and Malfreyt P 2009 Langmuir 25 9164-72

[19] Cossaro A et al 2008 Science $321943-6$

[20] Gannon G, Greer J C, Larsson J A and Thompson D 2010 ACS Nano 4 921-32

[21] Zhang Y, Barnes G L, Yan T and Hase W L 2010 Phys. Chem. Chem. Phys. 12 4435-45

[22] Nuzzo R G, Dubois L H and Allara D L 1990 J. Am. Chem. Soc. 112 558-69

[23] Lenk T J, Hallmark V M, Hoffmann C L, Rabolt J F, Castner D G, Erdelen C and Ringsdorf H 1994 Langmuir 10 4610-7

[24] Clegg R S and Hutchison J E 1996 Langmuir 12 5239-43

[25] Angelova P N, Hinrichs K, Philipova I L, Kostova K V and Tsankov D T 2010 J. Phys. Chem. C 114 1253-9

[26] Tamchang S W, Biebuyck H A, Whitesides G M, Jeon N and Nuzzo R G 1995 Langmuir 11 4371-82

[27] Templeton A C, Chen S W, Gross S M and Murray R W 1999 Langmuir 15 66-76

[28] Pirrung M C 2002 Angew. Chem. Int. Edn 41 1276-89

[29] Halliwell C M and Cass A E G 2001 Anal. Chem. 73 2476-83

[30] Kurth D G and Bein T 1993 Langmuir 9 2965-73

[31] Pallavicini P, Dacarro G, Galli M and Patrini M 2009 J. Colloid Interface Sci. 332 432-8

[32] Huang H, Tang C, Zeng Y, Yu X, Liao B, Xia X, Yi P and Chu P K 2009 Colloids Surf. B 71 96-101

[33] Ferhan A R, Guo L and Kim D-H 2010 Langmuir 26 12433-42

[34] Perez-Juste J, Pastoriza-Santos I, Liz-Marzan L M and Mulvaney P 2005 Coord. Chem. Rev. 249 1870-901 